\title{
Research on Hysteretic Performance of Q690 High Strength Steel High-Web Box-Section Steel Column
}

\author{
Jianpeng Sun ${ }^{1}$, Wei Feng ${ }^{2}$, RuiPeng Guo ${ }^{2}$, Chunfeng Liu $^{2}$ \\ ${ }^{1}$ Qingdao University of Technology, School of Civil Engineering, 266033 Qingdao, China \\ ${ }^{2}$ Shandong Hi-speed Engineering Construction Croup Co.Ltd., Jinan 250014, China
}

\begin{abstract}
In this paper, the finite element simulation software ABAQUS was used to study the hysteretic performance of Q690 high-strength steel (HSS) and high-web box-section steel columns. The finite element model was established by solid elements, and the influence of the initial defects of materials on the specimen was considered. The hyteretic performance of the specimen was conducted by analyzing and comparing the width-thickness ration of the flanges and the width-thickness ration of the webs. The results show that the increase of width-thickness ratios of the webs and flanges will reduce the hysteresis performance, the energy dissipation capacity and the ultimate horizontal bearing capacity of the specimen.
\end{abstract}

\section{Introduction}

High-strength steel (HSS) generally refers to steel with a yield strength of no less than $460 \mathrm{MPa}$. It exerts advantages in structural stress and can improve the safety and structural reliability of components. What's more, under the same load, the cross section of the component using HSS is usually smaller than that using ordinary steel, and thus the weight of the component is reduced. At present, high-strength steel has been successfully applied in many engineering projects such as building structures, bridge construction, and largespan high-rise structures. Considering the effect of earthquake on high-strength steel components, domestic and foreign scholars have carried out a lot of researches on the hysteretic behavior of HSS pressure-bending components.

In an early study, Kuwamura [1] pointed out that components made of HSS can be used in seismic design. Li Guoqiang et al. [2] have carried out experimental research and finite element analysis on hysteretic performance of welded cross-section pressure-bending members made of Q460C domestic HSS. Experiments show that when the width-thickness raito is below the requirement of "first-class seismic resistance", there will be plastic hinges at the bottom of the component, and local buckling will occur on the specimen as the widththickness ratio increases. The analytic results by using ANSYS finite element simulation software are in good agreement with the experimental measurement results. Shi Gang et al. [3, 4] conducted experimental research on the hysteretic performance of Q460 HSS box-section steel columns and Q460 HSS I-section steel columns, and analyzed and compared the effects of several experiment factors on specimens. Studies have shown that as the width-thickness ration off the web, the specimen is more prone to local buckling, and the ratio of the position of the maximum local deformation to the height of the specimen will also decrease accordingly. Chen Suwen et al. [5,6] studied the hysteretic performance of Q690 HSS components by using two Q690D HSS welded box-steel columns and two Q690D HSS welded I-shaped steel columns, and suggested to strictly implement flame correction during the manufacturing of HSS components to ensure its mechanical performance. The ANSYS finite element simulation results, combined with parameter analysis, show that the increase of length-slenderness ratio, axialcompression ratio and width-thickness ratio will cause a quick local deformation to the specimen and will affect the plastic development of the specimen.

Previous researches on the hysteretic performance of Q690 HSS component focused on compression-flexure members with low web. In this research, a Q690 HSS box-section steel column with high web sections is taken as the target. Such members have a higher ultimate bearing capacity, which is helpful for anti-seismic structure. Furthermore, the influences of the widththickness ratio of flanges and the width-thickness ratio of webs on the hysteretic performance will be compared and analyzed.

\section{Specimen design}

In order to study the hysteretic performance of high-web box-section frame columns made of Q690 HSS, the frame column is simplified as a cantilever column with one end fixed and one end unfixed. The height range of the component is selected from the bottom end of the frame column to the point of the column reflex. The

\footnotetext{
* Corresponding author: tang.lei2018@outlook.com
} 
schematic diagram of the component force is shown in Figure 1.

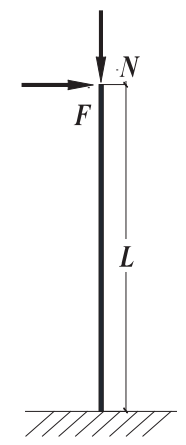

Fig 1 Force of cantilever column

The dimensions of the Q690 HSS high-web boxsection steel column are shown in Table 1, where H, B, $\mathrm{h} 0, \mathrm{~b}, \mathrm{tw}, \mathrm{tf}$ represent the cross-section parameters of the specimen, as shown in Figure 2, and L represents the height of the specimen. Taking specimen H-12-8-1 as an example, $\mathrm{H}$ represents HSS, 12 represents the thickness of the flange, 8 represents the thickness of the web, and 1 represents the axial-compression ratio of the specimen is 0.1 ( 2 means that the axial-compression ratio of the specimen is 0.2 )

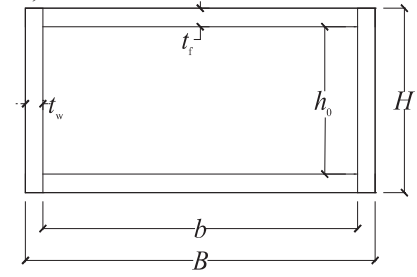

Fig. 2. Sectional parameters of specime

Table 1. Geometric dimensions of specimen

\begin{tabular}{cccccccc}
\hline Specimen & $H / \mathrm{mm}$ & $B / \mathrm{mm}$ & $h_{0} / \mathrm{mm}$ & $b / \mathrm{mm}$ & $t_{\mathrm{w}} / \mathrm{mm}$ & $t_{\mathrm{f}} / \mathrm{mm}$ & $L / \mathrm{mm}$ \\
\hline H-8-8-1 & 200 & 400 & 184 & 384 & 8 & 8 & 1210 \\
H-8-8-2 & 200 & 400 & 184 & 384 & 8 & 8 & 1210 \\
H-12-8-1 & 200 & 400 & 176 & 384 & 8 & 12 & 1210 \\
H-12-8-2 & 200 & 400 & 176 & 384 & 8 & 12 & 1210 \\
H-8-12-1 & 200 & 400 & 184 & 376 & 12 & 8 & 1210 \\
H-8-12-2 & 200 & 400 & 184 & 376 & 12 & 8 & 1210 \\
H-12-12-1 & 200 & 400 & 176 & 376 & 12 & 12 & 1210 \\
H-12-12-2 & 200 & 400 & 176 & 376 & 12 & 12 & 1210 \\
\hline
\end{tabular}

\section{Finite element model}

ABAQUS is used to perform finite element simulation on the designed specimen. The model is created with solid elements, and coupling constraints are used to impose boundary conditions on both ends of the specimen. The grid the specimen is uniformly divided, and the seed spacing is set to be $30 \mathrm{~mm}$, as shown in Figure 3.

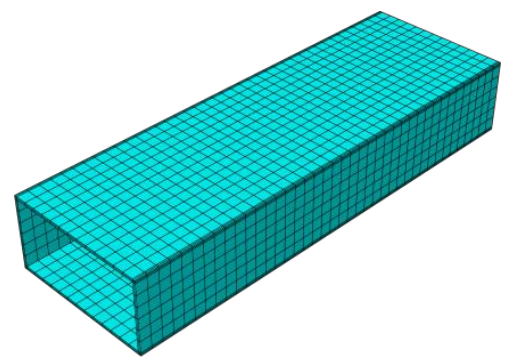

Fig 3 Grid division of specimen

The steel adopts a multi-linear isotropic strengthening model, and the stress-strain relationship curve is shown in Figure 4. Among them, the elastic modulus of the steel $\mathrm{E}=206 \mathrm{GPa}$, the yield strength fy $=620 \mathrm{MPa}$, the tensile strength $\mathrm{fu}=725 \mathrm{MPa}$, the yield ratio $\mathrm{fy} / \mathrm{fu}=0.86$, and the ultimate strain $\varepsilon \mathrm{u}=0.0592$.

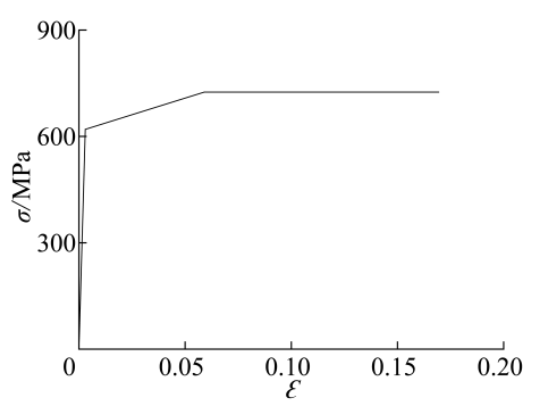

Fig 4 Stress-strain relationship of Q690 HSS

In the simulation experiment, both force loading and displacement loading are applied. Force loading is applied in the vertical direction, and the axial force is exerted at the coupling point of the top of the column according to the designed axial-compression ratio $n$. Displacement loading is applied in horizontal direction. According to the displacement angle limits of the elastic interlayer in frequent earthquake region and the displacement angle limits of elastoplastic interlayer in rare earthquake region required in GB50011-2010 Code for Seismic Design of Buildings, the displacement loading rule is shown in Table 2 and the displacement of each stage is cycled twice. 
Table 2. Displacement loading system

\begin{tabular}{cccccccc}
\hline $\begin{array}{c}\text { Loading } \\
\text { grade }\end{array}$ & 1 & 2 & 3 & 4 & 5 & 6 & 7 \\
\hline $\begin{array}{c}\text { Loading } \\
\text { displacemen }\end{array}$ & $\pm L / 300$ & $\pm L / 150$ & $\pm L / 100$ & $\pm L / 50$ & $\pm L / 40$ & $\pm L / 30$ & $\pm L / 20$ \\
\hline
\end{tabular}

\section{Result analysis}

There is outward buckling failure at the bottom end of the Q690 HSS high-web box-section steel column in the final stage of loading, and the distance between the maximum deformation position and the fixed end of the column was about $\mathrm{H} / 2$. The situations of $\mathrm{H}-8-8-1$ and $\mathrm{H}-$ 12-8-1 are shown in Figure 5.

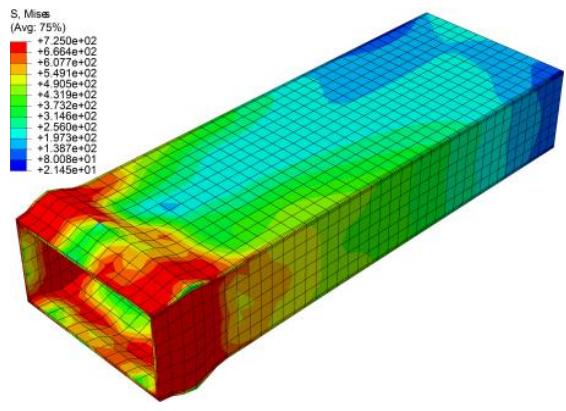

(a) Specimen H-8-8-1

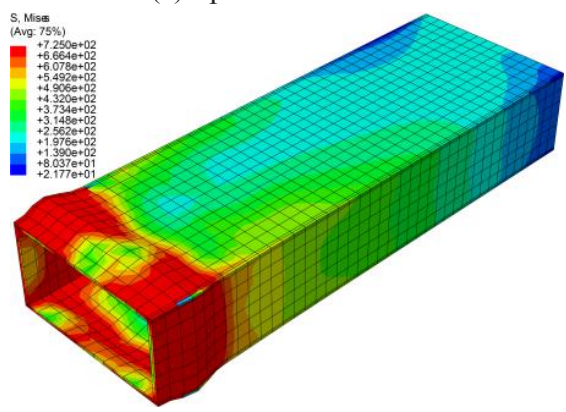

(b) Specimen H-12-8-1

Fig. 5. Deformation diagram of the specimens

Extract the hysteresis curve of each specimen from ABAQUS, and compare and analyze the influence of width-thickness ratio of the flange. Figure 6 shows that as the width-to-thickness ratio of the flange decreases, the hysteresis curve becomes plumper, and the area covered by the weekly hysteresis curve becomes larger, indicating that reducing the flange's width-to-thickness ratio helps to improve the energy dissipation capacity of the component. The decrease of width-thickness ratio also helps to delay local buckling, and further improves the horizontal ultimate bearing capacity of the component.

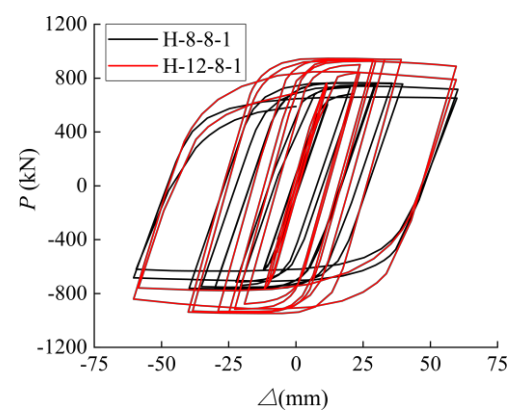

(a) Hysteresis curve

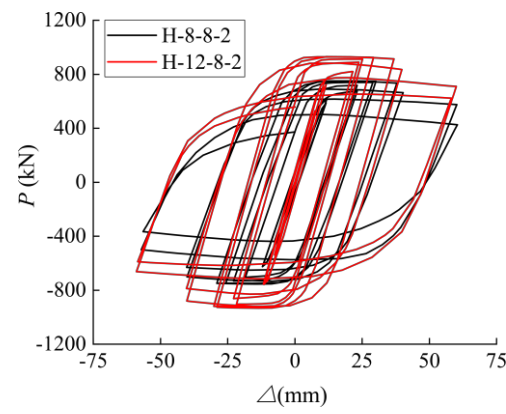

(b) Hysteresis curve

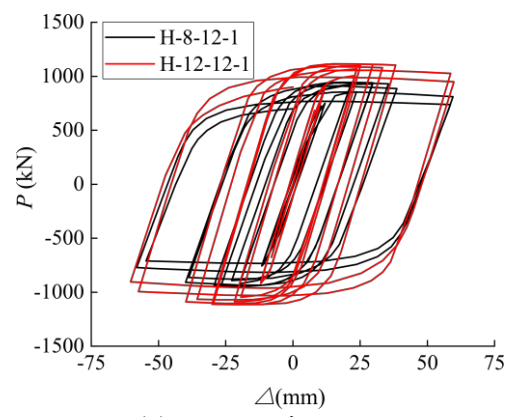

(c) Hysteresis curve

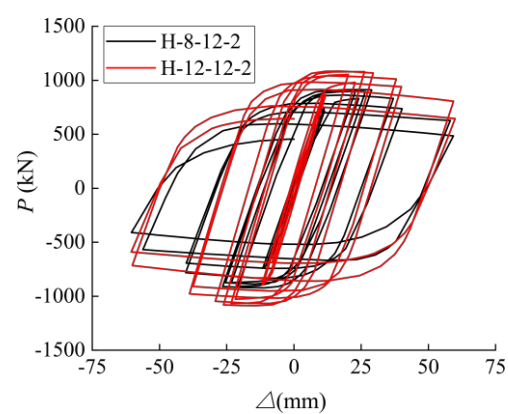

(d) Hysteresis curve

Fig. 6. Hysteresis curve 


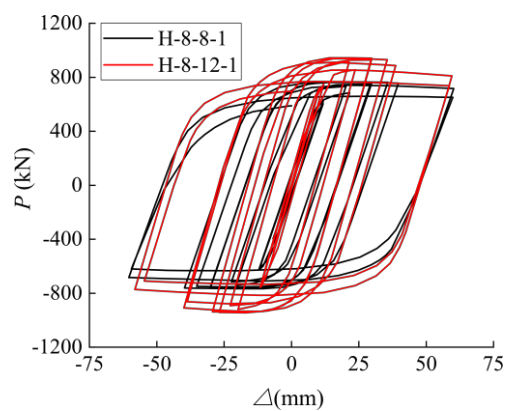

(a) Hysteresis curve

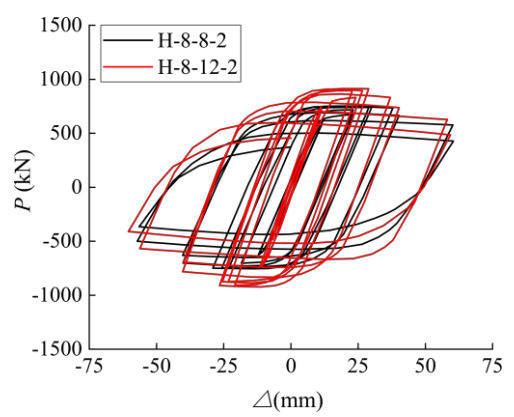

(b) Hysteresis curve

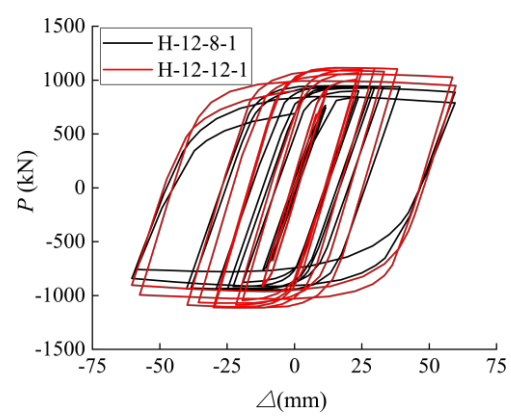

(c) Hysteresis curve

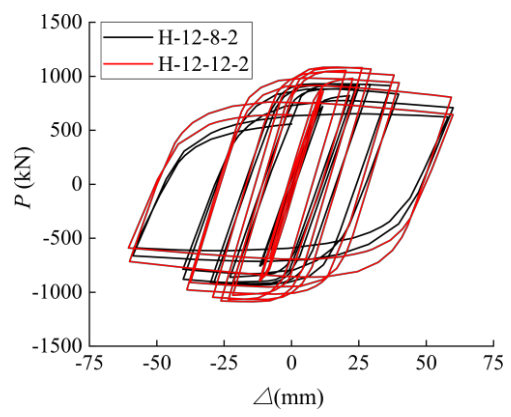

(d) Hysteresis curve

Fig. 7. Hysteresis curve

\section{Conclusions}

(1) The Q690 HSS high-web box-section steel column is damaged in the form of local buckling at the bottom of the column under constant axial horizontal cyclic loading. Local buckling hinders the ductility development of the component and is not conducive to the seismic resistance.
(2) The reduction of the flange's width-thickness ratio and the web's width-thickness ratio helps to improve the energy dissipation performance and the horizontal ultimate bearing capacity of the component. Compared with increasing the web's width-thickness ratio, the increase of the flange's width-thickness ratio has a greater effect on improving the hysteresis performance of the component.

(3) All the specimens have a good horizontal bearing capacity at L/50, which means that these specimens meet the requirements for displacement angel of the elastoplastic interlayer of multi-story steel structures in rare earthquake situation. Therefore, these specimens can be used in future engineering projects.

\section{References}

1. Kuwamura H, Kato B. Inelastic Behavior of High Strength Steel Members with Low Yield Ratio (1989). Proceedings of the 2nd Pacific Structural Steel Conference, 429-437.

2. Li, G.Q.,Wang, Y.B. (2013) Experimental study of Q460C high strength steel welded $\mathrm{H}$-section and box-section columns under cyclic loading. J. Build. Struc., 80-86.

3. Shi, G., Deng, C.S., Ban, H.Y. (2012) Experimental Investigation On Inelastic Behavior of Composite Box Girder Under Negative Moment. Int. Chin. Civil. Eng. J., 45: 53-61.

4. Shi, G., Deng, C.S., Ban, H.Y., Chen, Y.Y. (2012) Experimental study on hysteretic behavior of high strength steel box-section columns. J. Build. Struc., 33: 1-7.

5. Chen, S.W., Lu, Z.L., Li, G.Q., Wei, M., Wang, Y.B., Chen, X. (2014) Cyclic loading tests of Q690D high strength steel welded columns. J. Build. Struc., 35: 97-103.

6. Chen, S.W., Lu, Z.L., Chen, X., Li, G.Q. (2015) Hysteretic Behavior of Q690D High Strength Steel Box-Section Beam Columns. J. Tongji. Univ., 43: 1135-1143. 